\title{
Reflets
}

Revue ontaroise d'intervention sociale et communautaire

\section{S’alphabétiser en français dans un milieu anglophone : défi à relever pour l'intégration des francophones analphabètes sur le marché de l'emploi de Toronto}

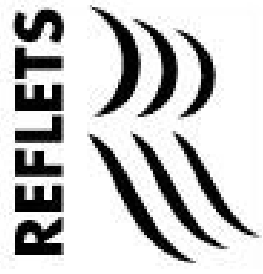

Myriam Odyssée Amenanka Asezo

Volume 6, numéro 2, automne 2000

Problèmes sociaux en Ontario français

URI : https://id.erudit.org/iderudit/026325ar

DOI : https://doi.org/10.7202/026325ar

Aller au sommaire du numéro

Éditeur(s)

Reflets : Revue ontaroise d'intervention sociale et communautaire

ISSN

1203-4576 (imprimé)

1712-8498 (numérique)

Découvrir la revue

Citer cet article

Odyssée Amenanka Asezo, M. (2000). S’alphabétiser en français dans un milieu anglophone : défi à relever pour l'intégration des francophones analphabètes sur le marché de l'emploi de Toronto. Reflets, 6(2), 221-225.

https://doi.org/10.7202/026325ar

Tous droits réservés (C) Reflets : Revue ontaroise d'intervention sociale et communautaire, 2000
Ce document est protégé par la loi sur le droit d'auteur. L'utilisation des services d'Érudit (y compris la reproduction) est assujettie à sa politique d'utilisation que vous pouvez consulter en ligne. 


\section{S'alphabétiser en français dans un milieu anglophone:}

\section{défi à relever pour l'intégration des francophones analphabètes sur le marché de l'emploi de Toronto}

\section{Myriam Odyssée Amenanka Asezo}

Centre Alpha-Toronto

Alphabétiser, c'est apprendre aux gens à lire, à écrire et à calculer. C'est aussi leur enseigner à sortir d'un monde fermé en leur donnant une nouvelle dimension, en les valorisant et en leur inculquant une nouvelle confiance en eux. C'est dans cet ordre d'idées que Micheline St-Cyr a créé le centre Alpha-Toronto, il y a de cela 11 ans, dans le but d'aider les analphabètes francophones de la ville à se munir du précieux don qu'est la capacité de lire, d'écrire et de calculer dans leur langue.

Subventionné par le gouvernement provincial de l'Ontario, le Centre Alpha-Toronto a pour mandat d'offrir une formation de base en écriture, en lecture et en calcul. Depuis sa fondation, Alpha-Toronto a formé plusieurs adultes d'origines diverses.

\section{La clientèle francophone en alphabétisation}

La clientèle francophone en alphabétisation est essentiellement immigrante, en majorité féminine et originaire de pays en guerre ou en crise politico-économique permanente. Venues de pays 
soumis à des dictatures ou en proie à des crises socio-économiques, ces personnes ont manqué soit des moyens financiers pour se scolariser soit de l'encadrement familial adéquat pour être encouragées à fréquenter l'école ou à compléter des études secondaires.

Vu sa faiblesse économique, il est compréhensible que cette clientèle, constituée en majorité de réfugiés politiques et de nouveaux arrivants, reçoive de l'aide sociale pour pallier ses problèmes d'établissement. La situation sous-économique des apprenants explique leur difficulté d'établir un horaire fixe et de le respecter.

Par ailleurs, en plus des quelques immigrants qui acceptent de suivre la formation en alphabétisation dispensée par le Centre, il convient de signaler la présence de Canadiens " de souche" analphabètes qui ne veulent pas se reconnaître comme tels devant l'apparente humiliation de fréquenter un centre d"alphabétisation. Ils sont pourtant nombreux dans le milieu francophone de Toronto les Canadiens « de souche " analphabètes qui ne savent ni lire, ni écrire, et qui tentent de le cacher.

\section{Zone et critères d'intervention}

Alpha-Toronto est l'unique centre d'alphabétisation francophone au service de l'ensemble des francophones analphabètes de la métropole. Eu égard à l'immensité du territoire, le Centre AlphaToronto se butte à un problème concret : recruter les clients potentiels éparpillés sur une si grande étendue.

En termes de critères d'intervention, il est à noter que nos clients sont des adultes de plus de 19 ans, originaires de pays ayant le français comme langue officielle. Ils ont un niveau de formation scolaire inférieur à la douzième année et parlent couramment le français. Ainsi, toute personne qui remplit ces conditions est en principe admise suite à une évaluation diagnostique démontrant ses besoins en alphabétisation. 


\section{Les services de Alpha-Toronto}

Les centres d'alphabétisation en général, et Alpha-Toronto en particulier, offrent des services de base pour lesquels ils sont subventionnés :

1. Le service d'information et d'aiguillage : ce service communique l'information générale et particulière liée au programme d'alphabétisation. C'est à partir d'entretiens téléphoniques que nous identifions les clients potentiels, que nous leur fixons des rendez-vous pour d'éventuelles évaluations diagnostiques ou que nous les dirigeons vers d'autres services appropriés à leurs besoins.

2. Le service d'évaluation : à ce niveau, il est question d'évaluer l'apprenant de façon diagnostique en vue de déterminer son niveau d'analphabétisme et de le classer selon les cinq niveaux de l'alphabétisation. D'autres évaluations, formative et sommative, s'adressent à l'apprenant tout au long de sa formation.

3. Le service de l'élaboration d'un plan de formation : l'une des spécificités de la formation en alphabétisation est le fait qu'elle soit plus individualisée, selon les besoins de chaque apprenant, qu'uniformisée. Bien qu'il y ait certains points communs au niveau des matières enseignées, nous considérons davantage les indicateurs de réussite retenus dans les plans de formation individuelle établis selon les évaluations diagnostiques. Ces plans de formation respectent le niveau des apprenants et réferent aux résultats d'apprentissage définis dans le manuel intitulé Comment utiliser les résultats d'apprentissage. Ce texte définit la démarche que doit suivre l'apprenant pour atteindre le but qu'il s'est assigné.

4. Le service de formation proprement dite : la formation est indissociable du plan de formation établi à la suite de l'évaluation initiale. À ce stade, nous enseignons des notions retenues comme indicatrices de réussite à atteindre en vue de réaliser les objectifs fixés dans le plan de formation. Cette formation est plus individualisée que commune à cause du caractère 
personnel des objectifs à atteindre. Elle est très flexible et se fait en petits groupes; elle est même souvent individuelle et vise l'employabilité comme but ultime.

5. Le service de suivi : celui-ci assure un contact avec les apprenants jusqu'à six mois après leur départ du Centre. Il nous permet d'apprécier le résultat de leur formation et, conséquemment, de rajuster nos services.

\section{Défis à relever}

\section{Pourquoi s'alphabétiser en français dans une ville anglophone? anglophe?}

Nul n'ignore que l'alphabétisme est l'une des conditions sine qua non de la modernité. L'éducation en général et l'alphabétisation en particulier sont des atouts considérables qui procurent à un individu une certaine ouverture d'esprit sur les plans tant social, intellectuel qu'économique; elles permettent aux personnes de se doter de certaines compétences en vue de leur développement intégral.

C'est ainsi que certains analphabètes francophones se décident à poursuivre et à compléter une formation de base leur permettant de mieux s'intégrer dans la communauté franco-torontoise et au marché du travail. Autrement dit,l'alphabétisation ouvre les portes de l'employabilité.

Telle est la question que d'aucuns pourraient se poser. Cependant, aussi controversé que cela puisse paraitre, il est important de dire qu'il est plus facile de s'alphabétiser dans sa langue maternelle ou officielle que dans une langue étrangère. Par langue maternelle, j'entends la langue véhiculée par le milieu dans lequel une personne a grandi.Ainsi, même s'ils vivent dans une ville largement 
anglophone, il s'avère utile pour les analphabètes francophones qui veulent apprendre à lire et à écrire de le faire d'abord en français. Bien plus, l'apprentissage du français n'est pas incompatible avec celui d'une langue seconde.

En outre, la formation en français est d'autant plus importante qu'il y a de plus en plus d'emplois bilingues à Toronto. La chance d'accéder à ces emplois s'accroît pour ceux de nos apprenants qui, en plus de la langue anglaise ambiante, amènent sur le marché de l'emploi un bagage linguistique amélioré par une formation dans notre Centre. Une preuve que le bilinguisme anglo-français progresse à Toronto est que notre Centre reçoit de plus en plus d'appels de personnes intéressées à des programmes de français langue seconde en vue de meilleures chances d'emploi. Par ailleurs, ces programmes sont en plein essor dans la ville.

\section{Conclusion}

Le français est l'une des deux langues officielles du Canada. La formation de base en français est une des clés du succès pour les analphabètes francophones qui veulent mieux s'intégrer tant dans leur communauté qu'au marché du travail de Toronto. La formation de base en français représente un atout additionnel nécessaire à la survie de la culture française au Canada, un outil de conservation de notre richesse culturelle pour nous, les francophones. Apprendre le français n'exclut évidemment pas l'apprentissage ou l'approfondissement des autres langues parlées à Toronto. Cet apprentissage devrait aller de pair avec l'acquisition d'habiletés de lecture et d'écriture dans la langue maternelle. 